\title{
BMJ Open Delivering safe and effective test-result communication, management and follow-up: a mixed-methods study protocol
}

Maria R Dahm, ${ }^{1}$ Andrew Georgiou, ${ }^{1}$ Johanna I Westbrook, ${ }^{1}$ David Greenfield, ${ }^{2}$ Andrea R Horvath, ${ }^{3,4}$ Denis Wakefield, ${ }^{3,5}$ Ling Li, ${ }^{1}$ Ken Hillman, ${ }^{6,7}$ Patrick Bolton, ${ }^{8}$ Anthony Brown, ${ }^{9,10}$ Graham Jones, ${ }^{3,11}$ Robert Herkes, ${ }^{12}$ Robert Lindeman, ${ }^{13}$ Michael Legg, ${ }^{14,15}$ Meredith Makeham, ${ }^{16}$ Daniel Moses, ${ }^{3,17}$ Dauda Badmus, ${ }^{1}$ Craig Campbell, ${ }^{1,4}$ Rae-Anne Hardie, ${ }^{1}$ Julie Li, ${ }^{1}$ Euan McCaughey, ${ }^{1,3,18}$ Gorkem Sezgin, ${ }^{1}$ Judith Thomas, ${ }^{1}$ Nasir Wabe ${ }^{1}$

To cite: Dahm MR, Georgiou A, Westbrook Jl, et al. Delivering safe and effective test-result communication, management and follow-up: a mixed-methods study protocol. BMJ Open 2018;8:e020235. doi:10.1136/ bmjopen-2017-020235

- Prepublication history for this paper is available online. To view these files, please visit the journal online (http://dx.doi org/10.1136/bmjopen-2017020235).

Received 23 October 2017 Accepted 3 November 2017

Check for updates

For numbered affiliations see end of article.

Correspondence to

Dr Maria R Dahm;

maria.dahm@mq.edu.au

\section{ABSTRACT}

Introduction The failure to follow-up pathology and medical imaging test results poses patient-safety risks which threaten the effectiveness, quality and safety of patient care. The objective of this project is to: (1) improve the effectiveness and safety of test-result management through the establishment of clear governance processes of communication, responsibility and accountability; (2) harness health information technology (IT) to inform and monitor test-result management; (3) enhance the contribution of consumers to the establishment of safe and effective test-result management systems.

Methods and analysis This convergent mixedmethods project triangulates three multistage studies at seven adult hospitals and one paediatric hospital in Australia. Study 1 adopts qualitative research approaches including semistructured interviews, focus groups and ethnographic observations to gain a better understanding of test-result communication and management practices in hospitals, and to identify patient-safety risks which require quality-improvement interventions. Study 2 analyses linked sets of routinely collected healthcare data to examine critical test-result thresholds and test-result notification processes. A controlled beforeand-after study across three emergency departments will measure the impact of interventions (including the use of IT) developed to improve the safety and quality of test-result communication and management processes. Study 3 adopts a consumer-driven approach, including semistructured interviews, and the convening of consumer-reference groups and community forums. The qualitative data will identify mechanisms to enhance the role of consumers in test-management governance processes, and inform the direction of the research and the interpretation of findings.

Ethics and dissemination Ethical approval has been granted by the South Eastern Sydney Local Health District Human Research Ethics Committee and Macquarie University. Findings will be disseminated in academic, industry and consumer journals, newsletters and conferences.

\section{Strengths and limitations of this study}

- The description of multiple processes used to manage test results across seven adult hospitals and a children's hospital will provide rich and unique comparative evidence about hospitals of different size, geographical location (rural, regional and metropolitan) and specialty.

- We will use an innovative data-driven approach to generate evidence about the impact of critical results notification thresholds on test-result followup processes and outcomes across different patient groups.

- We will ensure consumer engagement across all stages of the study from inception to dissemination, and capture experiences and opinions of a range of health consumer representatives and front-line patients.

- Our quantitative analysis is limited to data related to patients admitted to hospital. We will be able to monitor readmissions across participating hospital sites. There are some consequences of failure to follow-up test results (eg, mortality after discharge or readmission to hospitals not involved in the study) that we will not be able to measure.

- Qualitative evaluation methods are often conducted with smaller samples which means that findings may not be readily generalisable to larger populations. Nevertheless, qualitative research findings will provide context-rich insights that can expand the scientific knowledge base. Our mixed-methods approach allows for triangulation and draws on the strengths of qualitative and quantitative approaches.

\section{INTRODUCTION}

WHO, World Alliance for Patient Safety has identified poor test-result management as a high-priority patient-safety area. ${ }^{1}$ Systematic reviews in test-result follow-up have shown 
that pathology and imaging test results fail to be followed up for $20 \%-62 \%$ of inpatients, and for up to $75 \%$ of patients treated in an emergency department (ED). ${ }^{2}$ Other research from a Sydney hospital showed that tests ordered on the day of patient discharge accounted for $47 \%$ of missed test results, raising questions about the necessity and appropriateness of ordering tests whose results are most likely never reviewed. ${ }^{3}$ Poor test-result follow-up can have major consequences for quality of care, including missed diagnoses and suboptimal patient outcomes. ${ }^{4}$ The urgency of the problem was highlighted by the US Emergency Care Research Institute's 2017 report on patient-safety concerns for healthcare organisations. The report listed data-integrity failures associated with health information systems, poor care coordination across levels of care and test-result reporting problems among their top 10 patient-safety concerns for $2017 .^{5}$

Information technology (IT) has a key role to play in the communication and follow-up of test results. ${ }^{6}$ However, new models of test management supported by IT can only succeed if they are part of robust clinical governance processes which can underpin safe test management. ${ }^{7}$ Effective solutions must engage all stakeholders to arrive at decisions about who needs to receive the test results, how and when the results are communicated, and how they are acknowledged and acted on. ${ }^{8}$ Meeting these challenges requires the establishment of robust and resilient partnerships between managers, clinicians, pathology and medical imaging departments, and must include the involvement of consumers.

\section{The establishment of effective test-result management systems in hospitals}

Tackling the issue of test-result follow-up goes hand-inhand with the establishment of an integrated governance structure ensuring the safety of patient care. Effective clinical governance systems involve the clear delineation of responsibilities, communication and workforce accountability, along with systems to monitor progress and deal with any risks or impediments. ${ }^{9}$ Many clinicians describe existing test-result management systems as inefficient and chaotic. ${ }^{10}$ There is a lack of clarity about responsibilities and accountabilities of ordering versus treating clinicians for test-result follow-up, especially in relation to attending to time-critical results. ${ }^{811}$

\section{Clear guidelines and standards for test-result follow-up}

Pathology and medical imaging services perform a major role in the delivery of patient care by ensuring reliable and accurate results are delivered in a timely fashion to inform clinical management decisions. ${ }^{1}$ One of the main sources of problems is errors in the reporting phase of the laboratory process, when test-result information is communicated to the requesting (or referring) clinician. Such errors are often due to a lack of agreed standards or guidelines among laboratories, medical imaging departments, hospitals and other healthcare settings about what are critical, unexpected or significantly abnormal results, and when and how these results should be communicated to the responsible clinician. ${ }^{12}{ }^{13}$ Definitions of thresholds for critical tests and subsequent notification processes for critical pathology and imaging test results are largely based on consensus and expert opinions, and lack a rigorous evidence base. ${ }^{12}$ Good practice recommendations in this area emphasise the importance of clear definitions of key terms, the need for agreed alert thresholds and time frames, and specified procedures for fail-safe communication of test results that pose a critical or significant risk to patient safety. ${ }^{81213}$ This lack of standards is currently under review by a collaboration between the Royal College of Pathologists of Australasia (RCPA) and the Australian Association of Clinical Biochemists (AACB) which has established the Pathology Information, Terminology and Units Standardisation programme. ${ }^{14}$ This new initiative aims to draw on existing evidence from pathology datasets to establish standardised pathology information structures and terminologies to improve recording, decision support and communication of laboratory information.

\section{Harnessing IT}

Several electronic applications have been developed to support test-result management processes. These include systems that can track pending test results at hospital discharge ${ }^{15}$ deliver result alerts to clinicians, act as safety nets in result notification ${ }^{16}$ or use tracking systems to document acknowledgement and clinical actions. ${ }^{6}$ Intuitively, one would have expected the development of electronic systems to overcome problems associated with the existence of hospital data silos, yet poor integration of electronic systems has emerged as one of the most significant hazards to patient safety in Australia and internationally. ${ }^{17}$ At any one point in time, most hospitals are unable to identify critical test results which have not been reviewed unless they conduct time-consuming and cumbersome audits involving paper (and electronic) records. ${ }^{18}$ In such cases, the identification of missed test results may well be too late to have any positive effect on patient safety. ${ }^{2}$ However, performing sophisticated data linkage provides opportunities for leveraging the vast quantities of information held in these existing datasets to support the monitoring of test-result follow-up, derive new evidence to establish clinically meaningful critical test-result thresholds and to identify the consequences of test-result notification practices. ${ }^{16}$ Sophisticated models developed for linking previously fragmented hospital databases within and across multiple hospitals have been used to successfully monitor key indicators of hospital performance, including test-ordering patterns, test-result turnaround times and to investigate the relationships between testing patterns and patient outcome measures such as patient length of stay and mortality. ${ }^{19}$

\section{Enhancing the contribution of consumers in the test- management process}

Failure to inform patients of their test results can have devastating consequences for their health ${ }^{4}$ and has been described as legally indefensible in malpractice claims. ${ }^{20}$ 
Table 1 ED presentations for 2016 and provision of ICU services across study hospital sites in SES LHD and IS LHD

\begin{tabular}{lllll} 
SES LHD and SCHN & ED presentations 2016* & ICU & IS LHD & ED presentations 2016* ICU \\
\hline Prince of Wales Hospital & 54443 & $\checkmark$ & Shellharbour Hospital & 29479 \\
Royal Hospital for Women, Sydney & Not applicable & $\checkmark \dagger$ & Shoalhaven Hospital & 38039 \\
St George Hospital & 76228 & $\checkmark$ & Wollongong Hospital & 61348 \\
Sutherland Hospital & 50025 & $\checkmark$ & & $\checkmark$ \\
Sydney Children's Hospital & 36700 & $\checkmark$ & & 128899 \\
Total & 217396 & & & $\checkmark$
\end{tabular}

${ }^{*}$ Calculated using quarterly data of presentations to EDs (January-December 2016) via Bureau of Health Information interactive data portal Healthcare Observer. ${ }^{47}$

†Acute care service.

ED, emergency department; ICU, intensive care unit; IS, Illawarra Shoalhaven; LHD, Local Health District; SCHN, Sydney Children's Hospital Network; SES, South Eastern Sydney.

Effective partnerships between patients and clinicians, and increased consumer engagement, have the reported benefits of making healthcare safer and better. ${ }^{21} 22$ This is relevant to test-result follow-up where patients can access their own appointment and personal information, and facilitate communication with health professionals by using secure electronic patient portals to access their electronic health records. ${ }^{23}$ However, major obstacles hinder the involvement of consumers, including a lack of educational tools and self-management guides outlining when it may be necessary to seek medical assistance. ${ }^{22}$ There is no consensus among clinicians regarding if, and when, patients should have access to their test results. ${ }^{24}$ Many clinicians report the need for consumer education and support tools if strategies such as the direct notification of results to consumers are instituted. ${ }^{25}$ This is contrasted by the views of many consumers who report they want access to their test results. ${ }^{26}$ It is crucial that consumers are involved in informing the development of any such strategies and advise on the type of tools which may be most effective and acceptable to a wide spectrum of consumers.

This National Health and Medical Research Council (NHMRC)-funded Partnership Project, undertaken in collaboration with the New South Wales (NSW) Health Pathology and the Australian Commission on Safety and Quality in Health Care (The Commission), will run from 2016 to 2020 and aims to make a significant contribution to enhanced patient safety in Australia and internationally. This innovative and comprehensive mixed-methods project combines quantitative and qualitative research across three studies to assess if the introduction of health IT incorporating clearly defined standards of communication, responsibility and accountability among pathology and medical imaging departments, hospital management and clinicians, and underpinned by consumer engagement will lead to improved safety and effectiveness of test-result follow-up practices.

The project's three aims will be addressed in three studies:

Aim 1: to improve the effectiveness and safety of test-result management through the establishment of clear processes of communication, responsibility and accountability (study 1);

Aim 2: to harness health IT to inform and monitor test-result management (study 2);

Aim 3: to enhance the contribution of consumers to the establishment of safe and effective test-result management systems (study 3 ).

\section{METHODS}

\section{Project design and setting}

We will conduct a convergent mixed-methods ${ }^{27}$ project over a 5-year period concurrently collecting qualitative and quantitative data for triangulation and corroboration. Integrating complementary qualitative and quantitative approaches will allow us to develop a more complete understanding of the complex issues surrounding test-result management and contribute to the evidence base to inform the development of interventions to enhance the safety and quality of test-result management.

The project will involve the Emergency Departments (EDs), intensive care units (ICUs) and general admission wards at eight hospitals in the Sydney Children's Hospital Network, South Eastern Sydney (SES) and Illawarra Shoalhaven (IS) Local Health Districts (LHDs) in NSW, Australia. In 2016, the study hospitals provided care to more than 340000 ED presentations. Table 1 provides an overview of all hospital sites for this project, the number of presentations to each ED in 2016 and provision of ICU services.

\section{Research plan}

Study 1

Aim: Improve the effectiveness and safety of test-result management through the establishment of clear processes of communication, responsibility and accountability.

We will adopt a problem-focused approach ${ }^{28}$ to map current test-management practices within our study sites, to develop an organisational test-result management clinical governance checklist and to implement improved test-management governance processes. 


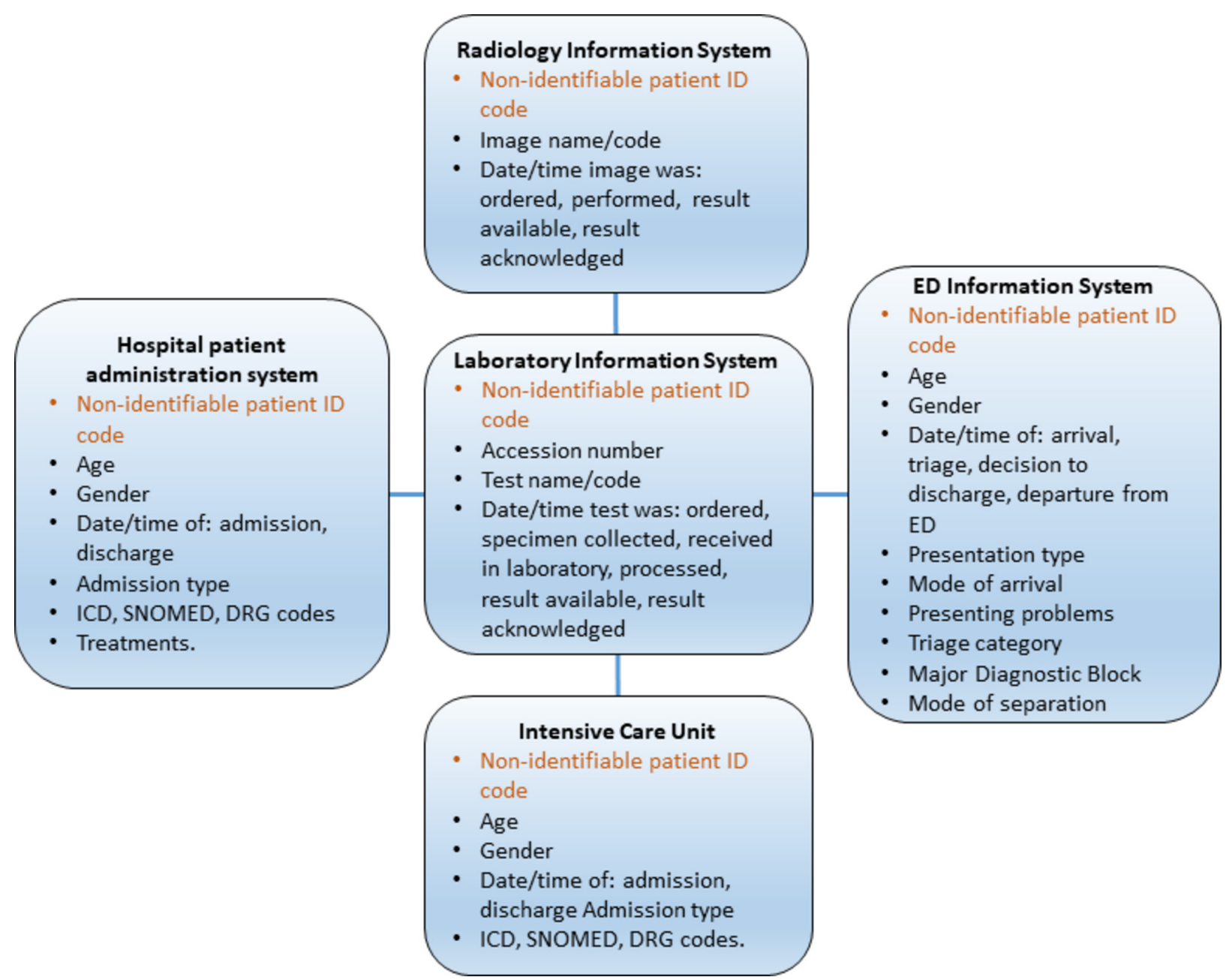

Figure 1 Data extraction and linkage outline. DRG, Diagnosis-related group; ED, emergency department; ICD, International Statistical Classification of Diseases and Related Health Problems; SNOMED, Systematized Nomenclature of Medicine.

To map test-management practices to outline current levels of communication, responsibility and accountability, the research team will use a range of qualitative appraisal techniques including audio-recorded focus groups, interviews with up to 10 clinical staff, and 'Think-Aloud' work observations with up to four clinical staff within each of the EDs, ICUs, general admission wards, pathology laboratories and medical imaging departments of the hospital study sites. Directors and nurse unit managers of the relevant departments at the study hospitals will be provided with introductory recruitment letters, along with participant information sheets and consent forms to be distributed to potential participants.

Depending on the hospital size, two members of the research team will be present at each site for a duration of 2-5 days, when clinical staff will be invited to participate in interviews. Interviews are expected to last between 20 and $30 \mathrm{~min}$, focus groups up to $40 \mathrm{~min}$. We anticipate that up to 50 interviews at each hospital site will be conducted for this part of the study (ie, 10 staff for each department) to capture all levels of staff across managerial and clinical hierarchies.
Questions for the semistructured interviews and focus groups will be exploratory, and are informed by current literature. They will cover aspects of test ordering, result notification and acknowledgement, delineation of abnormal versus critical versus significant results and communication of test results to patients, and within and across wards, and hospitals. ${ }^{8}{ }^{29}$ Multimodal data will be collected in the form of observational field notes, audio recordings, photographs and other relevant artefacts. All audio recordings will be de-identified and transcribed. We will employ triangulation of data, undertake participant validation via member checking and involve multiple researchers in qualitative coding to ensure the trustworthiness of the qualitative data. ${ }^{30}$ Transcripts will be analysed inductively for emerging themes ${ }^{31-33}$ by at least two members of the research team and analysed iteratively using NVivo qualitative data analysis software (QSR International, V.11, 2015). Qualitative data will be used to identify, map and compare current test-management work processes (eg, documenting the communication channels used for alerting doctors to critical test results) in the laboratories and different clinical environments across hospitals. ${ }^{33}$

Differences in test-management practices between hospitals will be identified, and adherence to national and 
Table 2 Power calculation for study to measure acknowledgement rate for critical test result

\section{Acknowledgement rate}

\begin{tabular}{|ll|}
\hline Before & $45.0 \%$ \\
\hline Percentage change & $+20.0 \%$ \\
\hline After & $54.0 \%$ \\
\hline $\begin{array}{l}\text { Number of critical tests to be reviewed per study } \\
\text { period for each arm at each site }(\mathrm{n})\end{array}$ & 700 \\
$\begin{array}{l}\text { Number of critical tests to be reviewed at each } \\
\text { site (2n) }\end{array}$ & 1400 \\
$\begin{array}{l}\text { Number of critical tests to be reviewed at three } \\
\text { ED sites during the study period }\end{array}$ & 4200 \\
\hline
\end{tabular}

ED, emergency department.

international recommendations and guidelines (where they exist) will be assessed to pinpoint evidence-practice gaps. These investigations will identify communication practices regarding:

- how test results of different levels of importance (eg, urgent, life threatening, critical but non-urgent results, etc) are communicated to clinicians in different settings (eg, ED vs ICUs);

- how responsibility is managed (eg, identification of who is sent the test result, escalation procedures when a responsible medical officer is unavailable);

- accountability structures (eg, organisational level procedures to identify test results which have not been reviewed across defined periods).

A test-result management clinical governance checklist will draw on empirical evidence and will be developed through an iterative consultation process with our stakeholders. Based on the findings of study 1 , we will draft a proposal checklist to be shared for comments (eg, to identify specific areas for intervention) with clinicians, laboratory and management staff at the hospitals during a series of workshops and with consumers in our community forum (see the Study 3 section). Stakeholder feedback will contribute to revisions of the checklist and stakeholders will be engaged in the approval of the checklist. The aim of the checklist will be to provide clear guidance to hospitals regarding the standardisation of test-management practices which will reduce the risk of failure to follow-up test results. Individual organisations will be able to compare existing processes against the checklist to identify areas requiring revision and/or change. The checklist is a change facilitation artefact that will serve a dual role-first, as a purpose-designed framework to direct the conduct of actions by the healthcare organisations, ${ }^{934}$ and second, as a translational research product which will be iteratively refined and developed for application in other healthcare settings (eg, general practice) across Australia and internationally.

Following on from the workshops, our research partners in NSW Health Pathology and across the participating hospitals will use the checklist to identify and deploy interventions aimed at improving the effectiveness and safety of test-result management within each organisation. Interventions may include the introduction of alert thresholds (relating to when a test result is considered urgent or critical) for a specified range of critical laboratory test results (as identified by our partners). Further intervention may involve implementation changes related to recommended modes of communication (eg, fax, pager, telephone or electronic alert) and clinical governance processes (eg, escalation process in cases where a doctor is not available).

\section{Study 2}

Aim: Harness health IT to inform and monitor test-result management.

Study 2 employs a data-driven approach and advanced data-linkage techniques using routinely collected data from healthcare databases to inform and monitor test-result management. A retrospective longitudinal study will be conducted to inform the adoption of critical test-result thresholds and notification processes (eg, using an algorithm to identify acute kidney injury (AKI)). A controlled before-and-after study across three EDs will be undertaken to measure the impact of interventions on the safety and quality of test results follow-up (eg, rates of result acknowledgement or rates of result return after discharge). Interventions include the clinical governance checklist developed in study 1 and additional possible intervention(s) implemented independently by NSW Health Pathology during the project period (eg, alert notification or clinical decision support systems).

\section{Data linkage}

Non-identifiable datasets from Laboratory Information System (LIS), hospital patient administration system (PAS), ED information system, ICU and Radiology Information System (RIS) will be provided to the research team to create a dataset linking patient demographics, their clinical information, test results and health outcomes. Figure 1 provides examples of specific fields extracted from each dataset, along with a schematic of the datalinkage process. Data will be rendered non-identifiable to the research team by staff within NSW Health Pathology, in such a way that a unique (quasi) patient ID will be generated which will be used to link different datasets.

The research team will conduct a data quality assessment and analyse the data for accuracy, comparability, completeness, conformity, consistency, relevance, timeliness, usability and validity. ${ }^{35}$ The initial dataset will cover the period from 1 January 2010 to 30 December 2016. An annual data extract will then be made at the end of June in each year of the study, to provide the research team with all patient information from the previous year.

\section{Retrospective longitudinal study}

To aid our understanding of the relationships between critical test-result thresholds, subsequent notification procedures, and a range of key process and outcome indicators, we will conduct a retrospective longitudinal study using 
routinely collected healthcare data and apply robust multilevel models. ${ }^{37}$ Working in consortium teams with our clinical and scientific partners and focused on specific patient groups (eg, patients undergoing tests such as potassium or serum creatinine), we will identify patient cohorts whose results fall within and outside existing definitions of critical test results (drawing on the definitions identified in study 1). For example, we will conduct a retrospective examination of laboratory identification of AKI which is acquired by approximately 13.3 million people globally, with mortality expected in $10 \%-15 \%$ of affected individuals. ${ }^{38}$ A lack of consensus around the definition of AKI has led to late identification and poor management of it leading to increased mortality, with hospital patients likely to be discharged with unrecognised AKI and others being managed inappropriately. ${ }^{39}{ }^{40}$ A standardised AKI algorithm was produced in the UK based on the serum creatinine changes described in the Kidney Disease: Improving Global Outcomes classification. ${ }^{39}$ This was followed by an electronic patient-safety alert across hospitals in England. ${ }^{39}$ We propose to undertake a retrospective cohort study of adult patients who were admitted to participating hospital sites between January 2010 and December 2013. The AKI cases will be identified by applying the UK laboratory-based algorithm to the linked data from laboratory and inpatient systems. The study will aim to: (1) determine the incidence of AKI applying a laboratory-based algorithm on the linked dataset, and (2) identify any AKI cases that may have been missed by comparing the algorithm findings to AKI status as recorded in the International Statistical Classification of Diseases and Related Health Problems, Tenth Revision, Australian Modification (ICD-10-AM) codes.

This and additional retrospective studies will answer questions such as whether different critical test thresholds (and the consequent notification processes, eg, direct phone call to a clinician vs passive alerting in the form of an asterisked result) have any significant association with a range of patient process or outcome indicators such as length of stay, readmission or mortality. These findings will inform interventions projected by NSW Health Pathology to be developed within the test-management governance checklist framework in study 1 , such as the introduction of new mobile or desktop technology to promote the notification and acknowledgement of test results or the development of context-specific frameworks for identification and notification of results based on critical risk, for example, abnormal, life-threatening and so on. We will then investigate differences in a range of process and outcome indicators for these patients, including time taken for notification of pending/completed/verified test results, time taken to access and acknowledge test results, retest rates, time to ED disposition decision, ED length of stay, mortality and readmission rates.

A previous project conducted in collaboration with NSW Health Pathology across six hospitals in SES and IS LHDs led to the development and extensive utilisation of a dataset linking patient demographics, their clinical information, test results and outcomes. This linked dataset incorporated some 2.8 million pathology tests (from the LIS), 147280 inpatient admissions (from PAS) and $176015 \mathrm{ED}$ presentations (from the ED information system, FirstNet). ${ }^{37}$ We anticipate a larger sample size for this study as we will incorporate additional data from ICUs andradiology information systems into the linked dataset.

\section{Controlled before-and-after study across three EDs}

To measure the effects of a range of possible test-result follow-up intervention(s) on care process and outcome indicators (to be decided in conjunction with our relevant stakeholders), we will conduct a controlled before-and-after study across three EDs, with two ED sites receiving the same intervention(s) and one control ED site. The study will measure the effects of interventions, eg, changes to critical test definitions, notification processes and changed modes of communication (eg, fax, pager, telephone or electronic alert) and clinical governance processes (eg, escalation process in cases where a doctor is not available), on the treatment of patients and their clinical outcomes. The study will concentrate on a set of laboratory results (eg, sodium, potassium, lactate, creatinine, magnesium) where the results are either: (1) critical and signify a pathophysiological state that is potentially life threatening, or (2) may result in significant patient morbidity, irreversible harm or mortality. The study will involve a retrospective clinical review of patient records carried out by two trained clinical practitioners (eg, nurses) over 2 months in years 3 and 5 of the project. The primary outcome measure will be the number of critical results reported, acknowledged and reviewed alongside the number where no acknowledgement is recorded. The study will also consider secondary outcome measures including: (1) the method by which these results were communicated to and acknowledged by ED medical practitioners, (2) the number of incidents of missed diagnosis and failure to act associated with unacknowledged (likely missed) test results, (3) the treatment (and time to treatment) associated with different critical test results, (4) the impact on patient outcomes eg, death, length of stay, discharge status (ie, transfer, admission to hospital or home) or re-presentation within 28 days.

A systematic review showed that the lack of follow-up of test results can reach up to $75 \%$ in EDs. ${ }^{2}$ Another study reported an acknowledgement rate of $45 \%$ for test results within critical values in EDs. ${ }^{42}$ Based on these data, we estimate that to detect a $20 \%$ change in the acknowledgement rate we require a sample size of 700 critical test results across each study period for each study site, and another 700 tests during each (2 months) study period for each control site, based on a two-sided test of proportions (alpha $=0.05$, power $=0.9$; table 2). Previous work examining test follow-up in the ED has confirmed the feasibility of this sample size. ${ }^{43}$

\section{Study 3}

Aim: Enhance the contribution of consumers to the establishment of safe and effective test-result management systems. 
This study adopts the philosophy of doing with rather than doing for, and is aimed at ensuring consumer involvement in all stages of the project from inception to dissemination. We sought advice from consumer representative organisations during the development of the research grant proposal (2014-2015). This collaboration focused on the role of patients in improving test-result management systems, and showed that the level of patient involvement in their care may be influenced by a number of factors, including health literacy levels, and access to appropriate tools, and educational and decision support aids. ${ }^{44}$

We will adopt three approaches to increase the opportunity for contribution by consumers, including both health consumer representatives and front-line patients, across all aspects of the qualitative and quantitative studies incorporated in this mixed-methods project, ranging from governance to shaping the research direction, interpretation of findings and the development of revised test-management governance processes. To this end, we will (1) conduct semistructured interviews with ED patients, (2) establish consumer reference groups (CRGs) and (3) conduct community forums.

\section{Semistructured interviews with ED patients}

To provide insights on test-result management processes and gain an additional consumer viewpoint, we will conduct semistructured interviews with adult patients accessing services in EDs across the study sites. Clinicians will identify eligible patients in the ED who underwent a pathology or imaging test. These patients will be approached and invited to participate in audio-recorded semistructured interviews. Interviews will be designed to cover their experiences regarding test-result management and result access during the current encounter, and more generally, to gauge their views about access to their personal health information in relation to test results. This will involve identifying the needs of patients to determine how pathology and medical imaging results are presented, what information is needed to ensure that results are easy to access and understand, and guide patient-centred decision-making. Semistructured interviews with $\sim 20$ participants at each selected site are anticipated to last $\sim 5 \mathrm{~min}$ and will be audio-recorded. Questions will be informed by current literature and updated in line with emerging findings from the other arms of the study. Transcripts of recordings will be used for thematic analysis. It is envisioned that consumers will be engaged in thematic analysis of these data. All qualitative data analysis in study 3 will be undertaken using the same software, methods and measures for trustworthiness outlined for study 1 .

\section{Consumer reference group}

To enhance the contribution of consumers to safe test-result management, including the direct transmission of test results to patients, we will establish a CRG which engages health consumer representatives in the governance structure and all research phases of the project.
Trained health consumer representatives for the CRG will be recruited via health consumer organisations (eg, Health Consumers NSW and LHDs) and include other key stakeholders (eg, Clinical Excellence Commission, Royal College of Pathologists of Australasia (RCPA). The CRG will provide feedback about the way that the study findings impact on aspects of patient care to help shape the direction of the research programme and inform the design of informational and educational tools to advance consumer engagement.

\section{Community forums}

To consider findings from the research and emerging issues concerning the safety and quality of care as related to test-result management (eg, possible impact of innovations such as the clinical governance checklist on work processes), members of the CRG will also be invited to participate in community forums to bring together 10 - 15 participants from major community stakeholders (including consumer groups, health professionals, IT vendors and healthcare organisations). A minimum of two forums will be held until project completion, and participants will be invited to discuss the research findings and help shape their translational capacity and diffusion across the healthcare community. Health consumer representatives will be reimbursed for their time at a rate of \$AUD40 (as per nominated reimbursement rates ${ }^{45}$ for each CRG meeting they attend. At CRG meetings, data will be collected in the form of observational field notes and audio recordings, and transcripts of recordings will be used for thematic analysis. This information will be used to include the consumer perspective in the interpretation of findings and identify direction for further research.

\section{EXPECTED OUTCOMES AND SIGNIFICANCE}

The translation of the findings from this study will help organisations address key issues related to the management and accountability of test-result management systems across all organisational levels. Our research engagement with our collaborating partners will generate evidence about the safety and effectiveness of major test-result follow-up interventions across laboratories, medical imaging departments and hospitals. The study will contribute to: (1) improved hospital care and health service delivery, (2) the development of healthcare guidelines to improve the delivery of safe and quality care, (3) improved end-user accessibility and involvement in health services and (4) increased health system capacity.

Study 1 will adopt a qualitative approach to map the test-result management and notification processes across eight study sites. This study will describe the multiple processes used to manage test results across seven adult hospitals and a children's hospital. Study 1 will produce a test-result management clinical governance checklist which represents a substantial contribution to the establishment of resilient governance structures that promote accountability in how test results are managed within and across healthcare settings. The checklist will help healthcare 
organisations achieve the best possible patient outcomes using governance systems that can track and monitor results and ensure that they are acted on when needed.

Study 2 will take a highly innovative data-driven approach to generate evidence regarding the relationships between critical test results (eg, results that reach a threshold defined as 'critical') and notification practices (eg, the time between the issuing of a critical result and when the clinician receives and/or views the result), and a range of patient process and outcome indications (eg, length of stay, mortality). Such an approach has not been undertaken previously on such a large scale for a range of tests and patient groups. The results of this aspect of the study will provide evidence across multiple hospitals about the effects of interventions on: (1) the number and type of critical results that are received, acknowledged and acted on; (2) the percentage of critical results and their thresholds which lead to specific actions and (3) the resultant impact on patient treatment and outcomes.

Study 3 is founded on the active involvement of consumers who will contribute to the design and implementation of major interventions (including eHealth applications) to enhance consumer engagement into the future. ${ }^{46}$ We will adopt multiple strategies to capture the experiences and opinions of trained health consumer representatives and ED patients covering all aspects and stages of the study from inception to dissemination, and from governance to interpretation of study findings.

\section{ETHICS AND DISSEMINATION}

The release of non-identifiable unit record data for research purposes has been approved by the Centre for Epidemiology and Evidence, NSW Ministry of Health.

All participants in study 1 and study 3 will provide written informed consent. These participants will not be asked for their identity. Identifying information that could be coincidentally revealed during interviews/focus groups will be de-identified during transcription or deleted from photographs and artefacts. The identity of participants will not be disclosed in the reporting of the results.

The privacy and identities of individuals described in the datasets used in study 2 will be protected by using a non-identifiable dataset. Only results aggregated at a high level will be reported. In the unlikely event of a result that describes a small number of individuals, statistical disclosure control methods (NSW Health, Privacy Issues and the Reporting of Small Numbers, September 2011) will be implemented to avoid the inadvertent inferential disclosure of individual identities. These methods include collapsing cells, cell suppression and cell modification, where appropriate. All reported results will meet a conservative minimum $\mathrm{k}>10$ individuals threshold limit for 'small cells'. While the dataset could potentially be reidentified, the coding used will be stored by NSW Health Pathology and will not be revealed to the researchers.

The project is funded under an NHMRC Partnership Project grant with local and national partners at clinical and policy levels. Funding partners include NSW Health Pathology, and the Australian Commission on Safety and Quality in Health Care. Other stakeholders include Health Consumers NSW, AACB, and RCPA. The study team includes academic researchers, hospital and laboratory clinicians, and senior policy-makers from national health and clinical agencies.

In the first instance, study findings will be reported at executive level at the hospital sites and to partners in a series of workshops to identify specific areas for intervention or to address any major safety or process issues related to test-result management. In addition, findings will be disseminated to a diverse audience through peer-reviewed academic literature, popular science communications, industry and consumer newsletters and journals, and at academic, industry and health consumer conferences. Given the diversity of the project team, further options for dissemination of results include translational and implementation strategies such as the inclusion of findings in the development of policy or health information systems.

\section{Author affiliations}

${ }^{1}$ Centre for Health Systems and Safety Research, Australian Institute of Health Innovation, Macquarie University, Sydney, New South Wales, Australia

${ }^{2}$ Australian Institute of Health Service Management, University of Tasmania, Sydney, New South Wales, Australia

${ }^{3}$ Faculty of Medicine, University of New South Wales, Kensington, New South Wales, Australia

${ }^{4}$ Clinical Chemistry and Endocrinology, Prince of Wales Hospital, NSW Health Pathology, Sydney, New South Wales, Australia

${ }^{5}$ Centre for Immunology, NSW Health Pathology, Sydney, New South Wales, Australia ${ }^{6}$ The Simpson Centre for Health Services Research, SWS Clinical School, University of New South Wales, Kensington, New South Wales, Australia

${ }^{7}$ Ingham Institute for Applied Medical Research, Liverpool, New South Wales, Australia

${ }^{8}$ School of Public Health and Community Medicine, Faculty of Medicine, University of New South Wales, Kensington, NSW, Australia

${ }^{9}$ Health Consumers New South Wales, Sydney, New South Wales, Australia

${ }^{10}$ Men's Health Information and Resource Centre, Western Sydney University,

Penrith, New South Wales, Australia

${ }^{11}$ SydPath, St Vincent's Hospital, Sydney, New South Wales, Australia

${ }^{12}$ Australian Commission on Safety and Quality in Health Care, Sydney, New South Wales, Australia

${ }^{13} \mathrm{NSW}$ Health Pathology, Chatswood, New South Wales, Australia

${ }^{14}$ Michael Legg \& Associates, Bulli, New South Wales, Australia

${ }^{15}$ Faculty of Engineering \& Information Science, University of Wollongong, Wollongong, New South Wales, Australia

${ }^{16}$ Australian Digital Health Agency, Sydney, New South Wales, Australia

${ }^{17}$ Medical Imaging Department, Prince of Wales Hospital, Randwick, New South Wales, Australia

${ }^{18}$ Neuroscience Research Australia (NeuRA), University of New South Wales, Kensington, New South Wales, Australia

Contributors AG, JIW, ARH, DG, LL and RL initiated the project and led the development of the National Health and Medical Research Council (NHMRC) grant proposal. AG, JIW, DG, ARH, DW, LL and KH are chief investigators on the project and all made contributions to the grant proposal and protocol in their specific areas of expertise. PB, AB, RH, GJ, ML, MM and DM are associate investigators on the NHMRC grant and provided input to the grant proposal and protocol, particularly 
in the areas of consumer engagement (AB) and standards for pathology reporting (GJ and ML). RL and RH are funding partners on the NHMRC partnership proposa and have made contributions in their areas of expertise, especially the use of health information system in pathology and patient safety policy related to test result management. DB, CC, MRD, R-AH, JL, EM, GS, JT and NW are members of the project team and contributed to the protocol in relation to data collection, interpretation of qualitative data and quantitative data linkage procedures. MRD prepared the first draft of this manuscript based on the grant proposal. All authors have reviewed and provided input.

Funding National Health and Medical Research Council, Partnership Project Grant number 1111925 .

\section{Competing interests None declared.}

Patient consent Obtained.

Ethics approval Ethics approval granted by the Human Research Ethics Committee of the South Eastern Sydney Local Health District (HREC/16/POWH/412), ratified by Macquarie University. All relevant site-specific assessments have been approved.

Provenance and peer review Not commissioned; peer reviewed for ethical and funding approval prior to submission.

Open Access This is an Open Access article distributed in accordance with the Creative Commons Attribution Non Commercial (CC BY-NC 4.0) license, which permits others to distribute, remix, adapt, build upon this work non-commercially, and license their derivative works on different terms, provided the original work is properly cited and the use is non-commercial. See: http://creativecommons.org/ licenses/by-nc/4.0/

(C) Article author(s) (or their employer(s) unless otherwise stated in the text of the article) 2018. All rights reserved. No commercial use is permitted unless otherwise expressly granted.

\section{REFERENCES}

1. Jha A. World alliance for patient safety - summary of the evidence on patient safety: implications for research. Geneva: World Health Organization, 2008:118. http://apps.who.int/iris/handle/10665/43874 (accessed 10 Oct 2017)

2. Callen J, Georgiou A, Li J, et al. The safety implications of missed test results for hospitalised patients: a systematic review. BMJ Qual Saf 2011;20:194-9.

3. Ong MS, Magrabi F, Jones G, et al. Last orders: follow-up of tests ordered on the day of hospital discharge. Arch Intern Med 2012;172:1347-9.

4. Clinical Excellence Commission. Clinical focus report: diagnostic tests - how access and follow-up affect patient outcomes. 2012 http://www.cec.health.nsw.gov.au/_data/assets/pdf_file/0007/ 259198/patient-safety-report-diagnostic-tests.pdf (accessed 10 Oct 2017)

5. ECRI Institute. Top 10 patient safety concerns for healthcare organizations. 2017 www.ecri.org/PatientSafetyTop10 (accessed 10 Oct 2017).

6. Schiff GD, Bates DW. Can electronic clinical documentation help prevent diagnostic errors? N Engl J Med 2010;362:1066-9.

7. Singh $\mathrm{H}$, Wilson L, Reis B, et al. Ten strategies to improve management of abnormal test result alerts in the electronic health record. J Patient Saf 2010;6:121-3.

8. Hanna D, Griswold P, Leape LL, et al. Communicating critical test results: safe practice recommendations. Jt Comm J Qual Patient Saf 2005;31:68-80

9. Greenfield D, Nugus P, Fairbrother G, et al. Applying and developing health service theory: an empirical study into clinical governance. Clinical Governance: An International Journal 2011;16:8-19.

10. Gandhi TK. Fumbled handoffs: one dropped ball after another. Ann Intern Med 2005;142:352-8.

11. Sherman $H$, Castro $G$, Fletcher $M$, et al. Towards an international classification for patient safety: the conceptual framework. Int J Qual Health Care 2009;21:2-8.

12. Campbell CA, Horvath AR. Harmonization of critical result management in laboratory medicine. Clin Chim Acta 2014;432:135-47.

13. Campbell $\mathrm{C}$, Horvath A. Towards harmonisation of critical laboratory result management - review of the literature and survey of australasian practices. Clin Biochem Rev 2012;33:149.

14. Pathology Information Units and Terminology Standardisation Project (PITUS). PITUS newsletter issue 1. 2013 https://www.rcpa.edu.au/
getattachment/8ea9dbba-35c9-4b13-b09d-fb2966413a07/PUTSUPDATE-Issue-1-Mar-2012.aspx (accessed 10 October 2017).

15. Dalal AK, Poon EG, Karson AS, et al. Lessons learned from implementation of a computerized application for pending tests at hospital discharge. J Hosp Med 2011;6:16-21.

16. Georgiou A, Lymer S, Forster M, et al. Lessons learned from the introduction of an electronic safety net to enhance test result management in an Australian mothers' hospital. J Am Med Inform Assoc 2014;21:1104-8.

17. Georgiou A, Prgomet M, Paoloni R, et al. The effect of computerized provider order entry systems on clinical care and work processes in emergency departments: a systematic review of the quantitative literature. Ann Emerg Med 2013;61:644-53.

18. Saleem JJ, Russ AL, Neddo A, et al. Paper persistence, workarounds, and communication breakdowns in computerized consultation management. Int J Med Inform 2011;80:466-79.

19. Georgiou A, Vecellio E, Toouli G, et al. The impact of the implementation of electonic ordering on hospital pathology services. Sydney: Australian Institute of Health Innovation, University of New South Wales, 2012.

20. Bolton P. A doctor's duty to follow up preventable conditions: young $v$ central australian aboriginal congress - a bridge too far? NTLJ 2012;2:154.

21. Australian Commission on Safety and Quality in Health Care (ACSQHC). National safety and quality health service standards. Sydney, Australia: Commonwealth of Australia, 2012.

22. The National Patient Safety Foundation. Safety is personal; partnering with patients and families for the safest care. $2014 \mathrm{http}: / /$ www.npsf.org/about-us/lucian-leape-institute-at-npsf/lli-reports-andstatements/safety-is-personal-partnering-with-patients-and-familiesfor-the-safest-care/ (accessed 10 Oct 2017).

23. Ammenwerth E, Schnell-Inderst $P$, Hoerbst $A$. The impact of electronic patient portals on patient care: a systematic review of controlled trials. J Med Internet Res 2012;14:e162.

24. Callen J, Georgiou A, Westbrook J. Evaluation of solutions aimed at reducingthe incidence of missed test results: can technology assist? Sydney, Australia: Asian Oceania Congress on Radiology, 2012.

25. Giardina TD, Callen J, Georgiou A, et al. Releasing test results directly to patients: a multisite survey of physician perspectives. Patient Educ Couns 2015;98:788-96.

26. Giardina TD, Modi V, Parrish DE, et al. The patient portal and abnormal test results: an exploratory study of patient experiences. Patient Exp J 2015;2:148-54.

27. Creswell JW, Plano Clark VL. Designing and conducting mixed methods research. 2nd ed. Los Angeles: Sage, 2011.

28. Royal College of General Practitioners (RACGP). Standards for general practices. 4th ed: RACGP, 2010.

29. Ash J, Singh H, Sittig DF. Test results reporting and follow-up SAFER guide. November 2016: The office of the national coordinator for health information technology. 2016 https://www.healthit.gov/safer/ guide/sg008

30. Patton MQ. Qualitative research \& evaluation methods: integrating theory and practice. Thousand Oaks, CA, USA: Sage Publications Inc, 2015

31. Corbin J, Strauss A. Basics of qualitative research: techniques and procedures for developing grounded theory. 4th ed. Los Angeles, CA, USA: SAGE Publications Inc, 2015.

32. Creswell JW. Qualitative inquiry and research design: choosing among five approaches. 3rd Edition. Los Angeles, CA, USA: Sage Publications Inc, 2013.

33. Westbrook Jl, Braithwaite J, Gibson K, et al. Use of information and communication technologies to support effective work practice innovation in the health sector: a multi-site study. BMC Health Serv Res 2009;9:201.

34. Greenfield D, Nugus P, Travaglia J, et al. Auditing an organization's interprofessional learning and interprofessional practice: the interprofessional praxis audit framework (IPAF). J Interprof Care 2010;24:436-49.

35. DAMA UK Data Quality Dimensions Working Group. The six primary dimensions for data quality assessment. $2013 \mathrm{http}: / /$ www. damauk. org/RWFilePub.php? \&cat $=403 \& d x=1 \& o b=3 \&$ rpn $=$ catviewleafpublic403\&id=106193 (accessed 10 Oct 2017).

36. Strome TL. Healthcare analytics for quality and performance improvement. Hoboken, NJ, USA: John Wiley \& Sons, Inc, 2013.

37. Li L, Georgiou A, Vecellio E, et al. The effect of laboratory testing on emergency department length of stay: a multihospital longitudinal study applying a cross-classified random-effect modeling approach. Acad Emerg Med 2015;22:38-46.

38. Lewington AJ, Cerdá J, Mehta RL. Raising awareness of acute kidney injury: a global perspective of a silent killer. Kidney Int 2013;84:457-67. 
39. Selby NM, Hill R, Fluck RJ. NHS England 'Think Kidneys' AKI Programme. Standardizing the early identification of acute kidney injury: the nhs england national patient safety alert. Nephron 2015;131:113-7.

40. Stewart J, Findlay G, Smith N, et al. Adding insult to injury: a review of the care of patients who died in hospital with a primary diagnosis of acute kidney injury (acute renal failure). London: National Confidential Enquiry into Patient Outcome and Death, 2009.

41. Georgiou A, Vecellio E, Toouli G, et al. Monitoring the impact of the electronic medical record on the quality of laboratory test ordering practices. In: Grain H, Schaper L, eds. Health informatics: digital health service delivery - the future is now!. Adelaide: IOS Press, 2013:33-8.

42. Kilpatrick ES, Holding S. Use of computer terminals on wards to access emergency test results: a retrospective audit. BMJ 2001;322:1101-3.
43. Callen J, Paoloni R, Georgiou A, et al. The rate of missed test results in an emergency department. Methods Inf Med 2010;49:37-43.

44. Georgiou A, Legg M, Edwards G. Patient access to pathology results: Pulse+IT, 2014

45. Health Consumer QLD. Remuneration and reimbursement of consumers. Position Statement 2015 http://www.hcq.org.au/wpcontent/uploads/2015/12/Consumer-Remuneration-Rates-Dec-2015. pdf (accessed 10 October 2017).

46. Hordern A, Georgiou A, Whetton S, et al. Consumer e-health: an overview of research evidence and implications for future policy. Him J 2011;40:6-14.

47. Bureau of Health Information. Interactive data portal: healthcare observer. 2017 http://www.bhi.nsw.gov.au/Healthcare_Observer (accessed 10 Oct 2017). 